\title{
Evaluación de parámetros seminales no convencionales en individuos cuyas parejas presentan muerte embrionaria temprana recurrente: en busca de un valor de referencia
}

\author{
Edisson Rodríguez¹, Aura María Gil-Villa', Daniel Camilo Aguirre-Acevedo², \\ Walter Cardona-Maya'1, Ángela P. Cadavid ${ }^{1}$ \\ 1 Grupo Reproducción, Universidad de Antioquia, Medellín, Colombia \\ 2 Instituto de Investigaciones Médicas, Universidad de Antioquia, Colombia
}

Introducción. En estudios previos se relacionaron las alteraciones en los parámetros espermáticos no convencionales con la presencia de muerte embrionaria temprana recurrente de la pareja; debido a esto, se planteó la necesidad de establecer un valor de referencia de utilidad clínica para el manejo de estos pacientes.

Objetivo. Evaluar los parámetros seminales convencionales y las pruebas funcionales de lipoperoxidación de las membranas espermáticas, capacidad antioxidante del plasma seminal e integridad de la cromatina espermática, en individuos cuyas parejas presentan muerte embrionaria temprana recurrente, con el fin de obtener un valor de referencia y poder identificar aquellos individuos en quienes la disminución de la fertilidad se pueda demostrar con estas pruebas.

Materiales y métodos. Se evaluaron los parámetros espermáticos convencionales y no convencionales de 47 muestras de semen, de las cuales, 36 pertenecían al grupo de individuos con disminución de la fertilidad por historia de muerte embrionaria temprana recurrente en sus parejas, y 11 muestras de individuos sanos con fertilidad reciente.

Resultados. Mediante el análisis discriminante se obtuvo una función que permitió clasificar los dos grupos analizados así: un valor menor de 0,50 para el 86,1 \% de los individuos del grupo de muerte embrionaria temprana recurrente y un valor mayor de 0,50 para clasificar el $81,8 \%$ de los individuos del grupo de fertilidad reciente.

Conclusiones. Este valor de referencia de 0,5, basado en los resultados de los análisis espermáticos y utilizando el análisis discriminante, permitiría categorizar a los pacientes que consulten por historia de muerte embrionaria temprana en sus parejas y le ayudaría al médico a sugerir un tratamiento más enfocado en la posible causa de la disminución de la fertilidad.

Palabras clave: espermatozoide, aborto espontáneo, estrés oxidativo, cromatina, antioxidantes, fertilidad.

Evaluation of atypical semen parameters in individuals whose couples had a history of early recurrent embryo death: in search for a reference value

Introduction. Previous studies related alterations in non-conventional seminal parameters with recurrent early embryonic death for one couple. A reference standard of clinical assessment is required for the management of these kinds of patients.

Objective. Normal semen parameters were established based on functional tests including lipid peroxidation of sperm membranes, antioxidant capacity of seminal plasma and integrity of sperm chromatin to compare with men whose partners have recurrent early embryonic death. These parameters set reference values to identify subfertile individuals whose condition can be attributed to altered semen parameters.

Materials and methods. The conventional and non-conventional semen parameters of 47 samples of semen were evaluated. Thirty-six samples were from subfertile individuals whose partners had a history of early recurrent embryo death, and 11 samples were from individuals with recent evidence of normal fertility.

Results. By discriminant analysis, the two groups were classified as follows: a value below 0.50 for $86.1 \%$ of individuals in the group of recurrent early embryonic death, and a value above 0.50 to classify $81.8 \%$ of individuals in the group of recent fertility.

Conclusions. This reference value of 0.5 based on the results of sperm tests can identify infertile male patients whose partners have a history of early embryonic death. This will aid the physician to suggest treatments more focused on the possible cause of subfertility.

Key words: Spermatozoa; spontaneous abortion; oxidative stress; chromatin; antioxidants; fertility. 
Las causas más relacionadas con los problemas masculinos de reproducción son los defectos de la función espermática, por la obvia relación de los espermatozoides con el proceso de fecundación. Sin embargo, el conocimiento de los factores o de las condiciones que provocan estas alteraciones funcionales espermáticas ha sido poco estudiado. Al estrés oxidativo le han atribuido algunas de estas alteraciones, debido al daño que se genera por un aumento en la producción de especies reactivas de oxígeno y a la poca capacidad antioxidante del plasma seminal para prevenir y corregir la noxa $(1,2)$. Se necesitan mínimas cantidades de especies reactivas del oxígeno en los procesos de fecundación y es así como se ha demostrado, en experimentos in vitro, que éstas tienen un papel importante en la capacitación, hiperactivación, reacción del acrosoma y fusión del espermatozoide con el oocito, además de su participación como segundos mensajeros mediante diferentes vías de activación intracelular $(1,3)$.

En condiciones fisiológicas, las especies reactivas del oxígeno en el semen son producidas en bajas cantidades por diferentes tipos de células, como los espermatozoides inmaduros y anormales, los leucocitos infiltrados y las células epiteliales (3). Se ha postulado que el aumento de las especies reactivas del oxígeno y la disminución de las defensas antioxidantes inducen daños en los ácidos nucleicos, las proteínas y los lípidos presentes en la membranas de los espermatozoides, y causan disminución de la movilidad y de la viabilidad y alteraciones en la pieza intermedia, los cuales finalmente producen cambios funcionales en las células espermáticas (4-6).

En los espermatozoides de hombres cuyas parejas tienen muerte embrionaria temprana recurrente, se ha observado un incremento significativo de aneuploidías, condensación anormal de la cromatina, fragmentación del ADN, apoptosis y morfología espermática anormal, al compararlos con espermatozoides de hombres fértiles (7-11). Recientemente, nuestro grupo propuso que el aumento en la lipoperoxidación de las membranas espermáticas y en la fragmentación del ADN, podría tener un papel importante en la muerte

Correspondencia:

Ángela P. Cadavid, Grupo Reproducción, Facultad de Medicina, Universidad de Antioquia, Medellín

Teléfonos: (574) 2196470 y 219 6476; fax: (574) 2191072

angelap.cadavid@gmail.com,reproducción@medicina.udea.edu.co

Recibido: 26/05/10; aceptado:12/11/10 embrionaria temprana recurrente (12-14). Incluso, se observó que después de implementar una dieta de alimentos o medicamentos que contenían antioxidantes, en seis individuos con historia de muerte embrionaria temprana recurrente en sus parejas y con alto porcentaje de fragmentación del ADN y aumento en la lipoperoxidación de las membranas espermáticas, cinco de ellas lograron finalizar exitosamente su embarazo (12).

El objetivo del presente estudio fue evaluar los parámetros seminales convencionales y las pruebas funcionales de lipoperoxidación de las membranas espermáticas, la capacidad antioxidante del plasma seminal y la integridad de la cromatina espermática de individuos cuyas parejas presentan muerte embrionaria temprana recurrente, con el fin de obtener un valor de referencia y poder identificar aquellos individuos en quienes se pueda demostrar la disminución de la fertilidad con estas pruebas.

\section{Materiales y métodos}

\section{Selección de pacientes y muestras de semen}

Se analizaron 47 muestras de semen, 34 de las cuales se habían presentado previamente (13). De estas muestras, 36 pertenecían a individuos cuyas parejas tenían historia de dos o más muertes embrionarias antes de la semana 12 de gestación, diagnosticada por medio de ultrasonido (grupo de muerte embrionaria temprana recurrente), y 11 muestras de individuos aparentemente sanos con fertilidad reciente (grupo control).

Las muestras de semen se obtuvieron mediante masturbación después de tres a cinco días de abstinencia sexual. Luego de completarse la licuefacción (30 a 60 minutos), se hizo el análisis seminal, siguiendo los lineamientos previamente establecidos por la Organización Mundial de la Salud en 1999 (15), mediante el cual se evaluó el volumen seminal y la concentración, la movilidad y la viabilidad espermática; además, la morfología se evaluó siguiendo los criterios estrictos de Kruger (16).

\section{Consideraciones éticas}

Este trabajo fue aprobado por el Comité de Ética para estudios en humanos de la Universidad de Antioquia. Cada individuo participó voluntariamente en el estudio y se obtuvo su consentimiento informado escrito antes de ingresar al estudio.

\section{Lipoperoxidación de las membranas}

Para evaluar la peroxidación lipídica de las membranas espermáticas producida por los radicales 
libres de oxígeno, se cuantificaron las sustancias reactivas al ácido tiobarbitúrico. El principio de esta técnica es que los peróxidos lipídicos son inestables y se descomponen para formar malondialdehído, que a temperaturas cercanas a $95^{\circ} \mathrm{C}$ y en presencia del ácido tiobarbitúrico, forma un aducto estable.

Brevemente, se colocaron $10^{7}$ espermatozoides resuspendidos en PBS y se incubaron durante una hora a $37{ }^{\circ} \mathrm{C}$ y con $5 \%$ de $\mathrm{CO}_{2}$. Luego, se mantuvieron en hielo durante 15 minutos y se agregó $0,5 \mathrm{ml}$ de ácido tricloroacético al $40 \%$ y $1 \mathrm{ml}$ de ácido tiobarbitúrico para precipitar las proteínas, y se calentó durante 15 minutos en baño seco. La solución se dejó enfriar a temperatura ambiente, se le agregaron $3 \mathrm{ml}$ de butanol y, finalmente, se centrifugó a $1.600 \mathrm{~g}$ durante 7 minutos.

La absorbancia se evaluó en un espectrofluorómetro (SpectraMax Gemini XS®, Molecular Devices, Downigtown, PA) a una longitud de excitación de $505 \mathrm{~nm}$ y de emisión de $546 \mathrm{~nm}$. Las sustancias reactivas al ácido tiobarbitúrico se expresaron en $\mathrm{nmol} / 10^{7}$ espermatozoides. El valor de referencia para muestras normozoospérmicas fue menor de $0,13 \mathrm{nmol} / 10^{7}$ espermatozoides (12).

\section{Estructura de la cromatina espermática}

La integridad de la cromatina espermática se evaluó por citometría de flujo con naranja de acridina. El principio de la técnica es que una cromatina condensada es menos sensible a condiciones de $\mathrm{pH}$ bajo, lo que permite que la naranja de acridina se intercale en el ADN de doble cadena y emita una fluorescencia verde. Por el contrario, cuando hay defectos en el empaquetamiento de la cromatina, el ADN se desnaturaliza en cadenas simples y las moléculas de naranja de acridina se unen al ADN y emiten una fluorescencia roja.

Para el procedimiento, las muestras de semen se diluyeron en solución tampón TNE [0,01 mol/L Tris-HCL, $0,15 \mathrm{~mol} / \mathrm{L} \mathrm{NaCl}$, y $1 \mathrm{mmol} / \mathrm{L}$ ácido etilen-diamino-tetraacético (EDTA), pH 7,4] a una concentración de $2 \times 10^{6}$ espermatozoides $/ \mathrm{ml}$. Se tomaron $200 \mu \mathrm{l}$ de la dilución inicial y se trataron con $400 \mu \mathrm{l}$ de una solución detergente ácida $(0,1$ $\%$ tritón X-100, 0,15 M NaCl, y $0,08 \mathrm{~N} \mathrm{HCl}$ a un $\mathrm{pH}$ de 1,2) durante 30 segundos. Posteriormente, se adicionaron $1,2 \mathrm{ml}$ de la solución colorante $(6 \mu \mathrm{g} /$ $\mathrm{ml}$ de naranja de acridina, $37 \mathrm{mM}$ de ácido cítrico, $126 \mathrm{mM}$ de $\mathrm{Na}_{2} \mathrm{HPO}_{4}, 1 \mathrm{mM}$ de EDTA disódica y $0,15 \mathrm{M}$ de $\mathrm{NaCl}, \mathrm{pH} 6,0$ ) y se leyó inmediatamente en un citómetro de flujo FACS $X L \AA$ (Coulter) recolectando entre 5.000 y 10.000 eventos. Además, una muestra de referencia fue corrida en cada ensayo como control.

El estado de la cromatina se analizó usando los resultados de la intensidad de fluorescencia media obtenida a partir del histograma, evaluando en FL1 la fluorescencia verde (ADN de cadena doble, nativo) y en FL3, la fluorescencia roja (ADN de cadena sencilla, desnaturalizado). Se determinó el índice de fragmentación del ADN (IFA) calculado con base en los valores obtenidos en el programa WinMD, así: IFA = intensidad de fluorescencia roja/intensidad de fluorescencia roja + verde, y se multiplicó por 100 para expresar el porcentaje de IFA, como había sido descrito previamente $(12,13)$.

\section{Capacidad antioxidante}

La capacidad antioxidante del plasma seminal se evaluó por el porcentaje de inhibición del radical estable 1,1-difenil-2-picril-hidracilo (DPPH) debido a su capacidad de donar hidrógenos. Para el procedimiento, las muestras de semen se centrifugaron a $660 \mathrm{~g}$ durante 8 minutos, se tomaron $200 \mu \mathrm{l}$ del sobrenadante y se agregaron a $4 \mathrm{ml}$ de la solución radical DPPH $\left(6,09 \times 10^{-5} \mathrm{~mol} / \mathrm{L}\right.$ metanol). La mezcla se incubó a temperatura ambiente durante 10 minutos para estabilizar la reacción de color y fue leída en un espectrofotómetro (Spectronic 20 Spectrophotometer $\circledast$; Genesys, Rochester, NY) a $515 \mathrm{~nm}$ contra un blanco de metanol; la muestra se leyó nuevamente, una hora después de la lectura inicial. Se utilizó una solución control de ácido ascórbico $(0,25 \mathrm{mg}$ de ácido ascórbico/1 $\mathrm{ml}$ de agua destilada) $(12,13)$.

\section{Análisis estadístico}

Las diferencias entre los grupos se evaluaron con el test de Mann-Whitney. Se consideraron diferencias estadísticamente significativas entre controles y pacientes, las que presentaran un valor $p$ menor de 0,05 . Este análisis se realizó con el programa estadístico Graph Pad Prism 5.0.

Además, con el fin de realizar una representación geométrica de la información obtenida de los parámetros espermáticos, se recurrió al método de ordenación multivariado de análisis de componentes principales. Este método intenta ordenar un conjunto de variables que pueden estar relacionadas o no estarlo y que expliquen la variación entre los datos en conjunto.

Así, considerando la variabilidad explicada por el comportamiento de los datos, resultaron dos componentes principales para este estudio, lo que 
permitió obtener un gráfico en dos dimensiones para visualizar datos y variables en un mismo espacio. El componente principal 1 (CP1) fue considerado como el eje principal, ya que presentó el más alto coeficiente de variabilidad dentro del sistema, con un valor de 39,2; a partir de este componente, se establecieron las relaciones entre las variables (parámetros espermáticos) y se realizaron todas las estimaciones estadísticas.

Cada uno de los cuadros representa un individuo y se obtiene de la suma de las variables del individuo, y los vectores representan las sumas de las variables en el grupo total de individuos. Las longitudes similares de los vectores en el gráfico sugieren la misma contribución de las variables en la distribución de los datos. Los ángulos de los vectores permitieron establecer las relaciones entre las resultantes, así: los ángulos de $90^{\circ}$ entre las variables indicaron que éstas no se correlacionaban; los ángulos mayores y menores de $90^{\circ}$ indicaban que la correlación era positiva o negativa; y, cuando el ángulo de la variable y el componente principal seleccionado como eje, es cercano a $90^{\circ}$, la variable no adquiere ningún peso en la correlación con las demás variables. Este análisis se realizó mediante el programa estadístico StatGraphic 5.0, utilizando un intervalo de confianza de $95 \%$, (figura 1 ).
Además, se hizo un análisis discriminante de los parámetros espermáticos evaluados; éste utiliza una función lineal similar a un análisis de regresión lineal múltiple, donde la variable dependiente es categórica y tiene como categorías la clasificación de cada uno de los grupos. Las variables independientes son continuas y determinan a qué grupos pertenecen los datos.

Mediante este análisis se pretende encontrar funciones lineales entre las variables continuas que discriminen mejor los grupos, lo que permite construir una regla de decisión sobre un objeto nuevo, que no se sabía cómo clasificar previamente, a uno de los grupos prefijados, con un mínimo grado de error (17).

La idea básica del análisis discriminante consiste en extraer una función basada en los coeficientes $\left(\mathrm{B}_{\mathrm{s}} \mathrm{s}\right)$ arrojados para cada variable, que permita diferenciar a los individuos con muerte embrionaria temprana recurrente que puedan deber la disminución de la fertilidad a la alteración de estas pruebas, o que, por el contrario, descarte la asociación con estos parámetros.

Se verificó el supuesto de que las matrices de varianzas y covarianzas son homogéneas en los grupos de estudio a partir de la prueba $\mathrm{M}$ de Box, en donde la hipótesis nula es de igualdad de

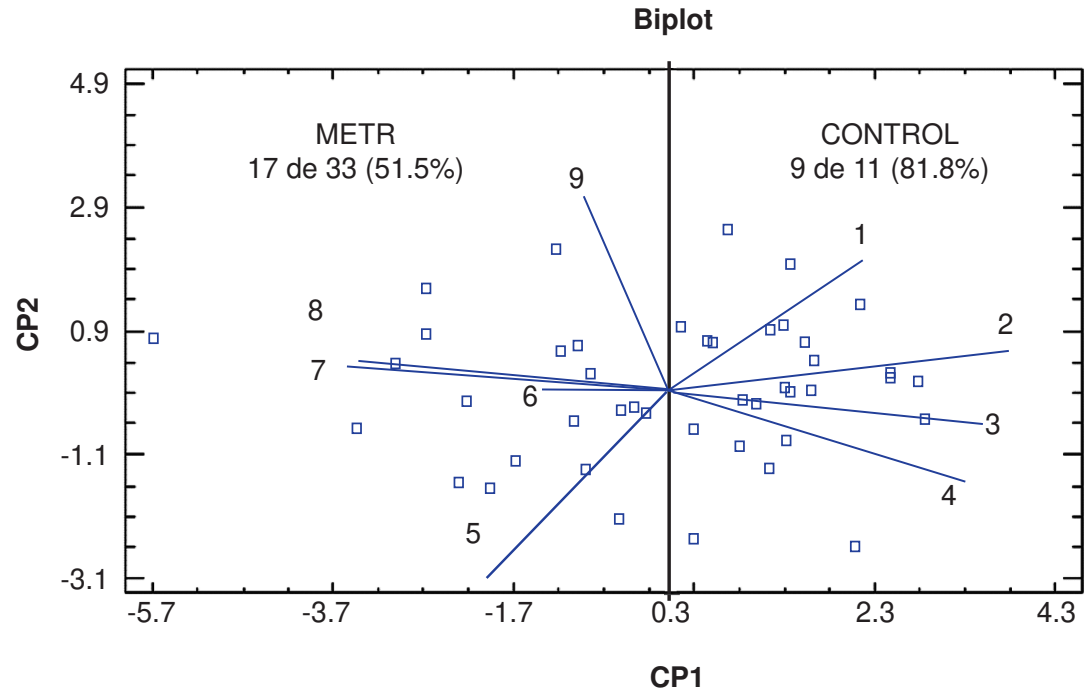

Figura 1. Análisis de componentes principales.

El componente principal 1 (CP1) divide el plano en dos. En el lado derecho se encuentran las variables: (1) capacidad antioxidante del plasma seminal, (2) movilidad progresiva, (3) morfología normal y (4) concentración espermática. En el lado izquierdo se encuentran las variables: (5) edad, (6) volumen del semen, (7) lipoperoxidación de las membranas espermáticas, (8) índice de teratozoospermia, y (9) fragmentación del ADN. Los individuos son representados por cuadrados; se encontró que 9 de 11 (81,8 $\%)$ individuos del grupo control se ubicaron en el lado derecho y que 17 de 33 (51,5\%) individuos del grupo de muerte embrionaria temprana recurrente se ubicaron en el lado izquierdo. 
matrices de varianzas y covarianzas. Se hizo un análisis por curvas ROC para establecer el punto de corte óptimo que maximizara la sensibilidad y la especificidad para determinar en cuál grupo, muerte embrionaria temprana recurrente o fértil, quedaría ubicado el paciente, a partir de los puntajes derivados de la función discriminante. Con el punto de corte obtenido, se calcularon los valores diagnósticos negativos, positivos y los cocientes de verosimilitud positivos y negativos. Estos análisis se hicieron con el software SPSS $17.0 \AA$.

\section{Resultados}

En este estudio se observaron diferencias estadísticamente significativas en la edad, la movilidad progresiva y el índice de teratozoospermia, al comparar los individuos del grupo de muerte embrionaria temprana recurrente con los individuos del grupo con fertilidad reciente $(p=0,0270 ; p=0,0170$; $p=0,0123$, respectivamente) (cuadro 1).

Al evaluar las correlaciones entre los parámetros espermáticos en todos los individuos, se encontró que existían correlaciones negativas estadísticamente significativas entre la producción de sustancias reactivas al ácido tiobarbitúrico y el índice de teratozoospermia contra la movilidad progresiva, la concentración espermática, el porcen-taje de morfología normal y la inhibición del radical DPPH.

Por otro lado, existían correlaciones positivas estadísticamente significativas entre la producción de sustancias reactivas al ácido tiobarbitúrico versus el índice de teratozoospermia y el volumen seminal; entre la concentración espermática y la movilidad progresiva, el porcentaje de morfología normal y el porcentaje de inhibición del radical DPPH. Se obtuvieron datos similares en resultados preliminares publicados por nuestro grupo (13).

El análisis de componentes principales mostró que el porcentaje de índice de fragmentación del ADN y la edad no se correlacionaba con las otras variables del CP1, debido a que formaban ángulos menores de $90^{\circ}$ con este componente. Las variables restantes contribuyeron de la misma manera en el análisis, a excepción del volumen, el cual fue representado por un vector más corto (figura 1).

Al dividir el plano del CP1 por la mitad, se observó que el $51,5 \%$ (17 de 33 individuos) del grupo de muerte embrionaria temprana recurrente se ubicó en el lado izquierdo y el 81,9\% (9 de 11 individuos) del grupo control se ubicó en el lado derecho del mismo componente (figura 1). Por su parte, la función discriminante obtenida cumplió el supuesto de que las matrices de varianzas y covarianzas eran homogéneas entre las poblaciones de estudio ( $\mathrm{M}$ de Box=75,68; F=1,056; gl1=45; gld2=1164,63; valor $p=0,373$ ).

El porcentaje de clasificación del grupo total usando la función discriminante fue de $85,1 \%$, la del grupo de muerte embrionaria temprana recurrente fue de $86,1 \%$ y la del grupo control fue de $81,8 \%$. Esta función discriminante puede escribirse como: $[-1,320+(0,002$ * edad $)+(0,362$ * volumen seminal $)$ + $(-0,011$ * concentración espermática $)+(0,038$ * movilidad progresiva $)+(0,052$ * morfología normal $)$ $+(-4,260$ * índice de teratozoospermia $)+(0,132$ *

Cuadro 1. Descripción general del grupo control y del grupo de muerte embrionaria temprana recurrente.

\begin{tabular}{lcccc}
\hline & Control $(\mathbf{n}=\mathbf{1 1})$ & METR $(\mathbf{n}=\mathbf{3 6})$ & Valor de $\boldsymbol{p}$ & Valor de referencia \\
\hline Edad (años) & $30 \pm 6,6(19-40)$ & $35,6 \pm 7,0(21-50)$ & $0,0270^{*}$ & $>2,0$ \\
Volumen (ml) & $4,5 \pm 2,1(2,4-10,2)$ & $3,7 \pm 1,6(1-8)$ & 0,0830 & $>20$ \\
Concentración $\left(10^{6} / \mathrm{ml}\right)$ & $57,6 \pm 19,9(24-90)$ & $55,0 \pm 34,2(5-130)$ & 0,6153 & $>14$ \\
Morfología normal (\%) & $20,5 \pm 7,1(7-28)$ & $17,2 \pm 5,6(31-5)$ & 0,1284 & $>50$ \\
Movilidad progresiva (\%) & $58,6 \pm 7,9(43-72)$ & $48,7 \pm 12,9(6-65)$ & $0,0170^{*}$ & $>1,6$ \\
Teratozoospermia (\%) & $1,3 \pm 0,1(1,2-1,7)$ & $1,5 \pm 0,2(1,2-1,7)$ & $0,0123^{*}$ & $<24$ \\
IFA (\%) & $18,5 \pm 4,2(14-26)$ & $16,3 \pm 5,0(5,7-32)$ & 0,1381 & $<0,13$ \\
SRATB (nmol/107 espermatozoides) & $0,08 \pm 0,05(0,03-0,17)$ & $0,11 \pm 0,1(0,02-0,51)$ & 0,4433 & $>35$ \\
Inhibición radical & & & & \\
DPPH (\%) & $50,3 \pm 13,3(28-75)$ & $40,9 \pm 13,4(14-64,6)$ & 0,0899 & \\
\hline
\end{tabular}

Las variables se representan como medias \pm desviación estándar y (rango)

* valor $\mathrm{P}<0,05$ control versus muerte embrionaria temprana recurrente

IFA: índice de fragmentación del ADN

SRATB: sustancias reactivas al ácido tiobarbitúrico

DPPH: 1,1-diphenil-2-picril hidracilo 
\%IFA $+(2,162$ * lipoperoxidación de membranas) $+(0,027$ * capacidad antioxidante $)]$; cada valor es multiplicado por el resultado obtenido en su respectiva variable y, finalmente, se hace una suma de todas las multiplicaciones.

Las curvas ROC permitieron establecer como punto de corte para los puntajes generados por la función discriminante, un valor de 0,50. Si el valor era inferior a 0,50 , el individuo sería clasificado en el grupo de muerte embrionaria temprana recurrente. El área bajo la curva fue 0,902 (IC95\% $0,76-1,00)$. Con este punto de corte se obtiene una sensibilidad de $86,11 \%$ (IC95 \% 73,43-98,80), especificidad de $81,8 \%$ (IC95 \% 54,48-100), valor diagnóstico positivo de 93,94\% (IC95\% 84,28-100), valor diagnóstico negativo de 64,29 (IC95 \% 76,60$89,76)$, cociente de verosimilitud positivo de 4,74 (IC95 \% 1,34-16,70) y cociente de verosimilitud negativo de 0,17 (IC95 \% 0,07-0,40).

$\mathrm{Si}$ al aplicar la fórmula del análisis discriminante a los resultados de los parámetros espermáticos se obtenía un valor superior a 0,5 , se consideraba que la disminución de la fertilidad del individuo no se debía a la alteración de estas pruebas, mientras que, si el valor era inferior a 0,50 , la disminución de la fertilidad sí podría relacionarse con la alteración de estos parámetros.

\section{Discusión}

Las especies reactivas del oxígeno han sido implicadas en infertilidad y en otros estados de enfermedad, como artritis, trastornos del tejido conjuntivo, enfermedad de Parkinson, sida, envejecimiento e infecciones (18). Específicamente en infertilidad, la generación excesiva de especies reactivas del oxígeno se asocia con daño celular espermático, defectos morfológicos, fragmentación del ADN, lipoperoxidación de las membranas, reacción del acrosoma disminuida y baja capacidad de fecundación (19).

La membrana celular de los espermatozoides es vulnerable al daño producido por las especies reactivas del oxígeno, debido a que ésta posee un alto contenido de ácidos grasos poliinsaturados que interactúan con metabolitos reactivos del oxígeno y provocan lipoperoxidación y desencadenan, además, sucesos que derivan en: alteraciones del retículo endoplásmico y de la mitocondria; rompimiento de las cadenas del ADN; liberación de enzimas celulares y finalmente, la muerte celular (20). Por estas razones, la peroxidación lipídica de la membrana se considera el mecanismo clave por medio del cual las especies reactivas del oxígeno provocan daños en el espermatozoide y conducen a infertilidad (20-22).

En estudios en los cuales los espermatozoides fueron expuestos a especies reactivas del oxígeno, producidas artificialmente, se observó un incremento significativo en el daño del ADN, posiblemente por la modificación de bases, deleciones, cambios en el marco de lectura, y alteraciones cromosómicas y de la integridad de la cromatina $(23,24)$. En otro estudio, en el cual se evaluó la fragmentación del ADN espermático en 24 hombres cuyas parejas presentaban pérdida de la gestación recurrente de causa no explicada, se encontró un incremento significativo en la morfología alterada y en la fragmentación del ADN espermático, lo que podría explicar la pérdida de la gestación en sus compañeras, pero no se halló correlación del daño del ADN con los parámetros seminales convencionales (25).

Khosrowbeygi et al. evaluaron los niveles de biomarcadores de estrés oxidativo en plasma seminal de individuos con alteraciones en los parámetros espermáticos y en un grupo control. Los resultados mostraron que la capacidad antioxidante total del plasma seminal era significativamente más baja, los niveles de superóxido dismutasa no variaron y los de 8-isoprostano libre fueron mucho más altos en los individuos con parámetros espermáticos alterados en comparación con los del grupo control. Los niveles de catalasa se correlacionaron positivamente con la movilidad y la morfología, y los niveles de 8-isoprostano se correlacionaron inversamente con estos mismos parámetros, lo cual permitió concluir que la disminución de la capacidad antioxidante total del plasma seminal podría estar relacionada con defectos de la función espermática (26).

En nuestro estudio, el análisis multivariado de componentes principales permitió establecer que la mayoría $(81,9 \%)$ de los individuos con fertilidad reciente tenían espermatozoides con alto porcentaje de formas normales, concentración espermática normal, alto porcentaje de movilidad progresiva y mejor capacidad antioxidante, y que el 51,5\% de los individuos del grupo de muerte embrionaria temprana recurrente tenían espermatozoides con alto índice de teratozoospermia y aumento de la producción de sustancias reactivas al ácido tiobarbitúrico, lo cual esta estaría de acuerdo con una causa multifactorial de la muerte embrionaria temprana recurrente. 
Por otro lado, el análisis discriminante permitió establecer una función utilizando todas las variables evaluadas y permitió una correcta clasificación de $81,8 \%$ para el grupo control y de $86,1 \%$ para el grupo de muerte embrionaria temprana recurrente. El valor de referencia de 0,5 podría modificarse si se requiere una mayor sensibilidad o especificidad para el análisis de individuos y esto permitiría ser más precisos en la detección de aquellos pacientes que puedan deber su infertilidad a la alteración de estas pruebas. Estos resultados sugieren que los individuos con un valor inferior a 0,50 presentan incremento en la producción de sustancias reactivas al ácido tiobarbitúrico y disminución de la capacidad antioxidante del plasma seminal, lo que podría asociarse a la muerte embrionaria temprana recurrente en sus parejas y se podría explicar por la relación que tiene el desequilibrio oxidativo con alteraciones de la morfología, la movilidad y la concentración espermática (11). Bajo estas condiciones, el espermatozoide podría fecundar el oocito; sin embargo, es posible que otros componentes celulares que son necesarios en el desarrollo embrionario estén alterados y, como consecuencia, este desarrollo no pueda darse adecuadamente $(12,13)$.

Por su parte, los individuos que estén relacionados con muerte embrionaria temprana recurrente y generen un valor superior a 0,50 , no deben la disminución de la fertilidad a la alteración de estas pruebas y la muerte embrionaria temprana recurrente en sus parejas podría tener otras causas.

La importancia de establecer este valor de referencia que permita identificar individuos con disminución de la fertilidad con muerte embrionaria temprana recurrente y alteración de estas pruebas, radica en que permitiría sugerir un tratamiento para los pacientes que neutralice la producción de especies reactivas del oxígeno (27) y mejore la capacidad antioxidante.

Los antioxidantes se han utilizado en tratamientos in vivo e in vitro debido a su capacidad protectora (27-28). Al incubar semen con una suspensión de neutrófilos activados, se crea un ambiente de estrés oxidativo, el cual lleva a la disminución de la movilidad progresiva, pero ésta se recupera al adicionar ciertos antioxidantes como la hipotaurina y la N-acetilcisteína, pero no al adicionar vitamina E (27). In vivo, se ha evaluado la capacidad de diferentes antioxidantes para proteger el espermatozoide humano (28); un estudio en el cual se realizó un tratamiento oral con vitamina $E$ y un suplemento de selenio durante tres meses, mostró mejoría notoria en la movilidad y una disminución significativa en la concentración de malondialdehído (28).

En conclusión, en el presente estudio se obtuvieron una función y un valor de referencia basado en la evaluación de la lipoperoxidación de las membranas espermáticas, la capacidad antioxidante del plasma seminal, la integridad de la cromatina espermática y los parámetros espermáticos convencionales, que permite clasificar a los individuos cuyas parejas tienen historia de muerte embrionaria temprana recurrente.

Sin embargo, estas pruebas son útiles para evaluar alteraciones del factor masculino como posible factor de riesgo para muerte embrionaria temprana recurrente, pero no se pueden considerar como marcadores definitivos del problema objeto de la investigación, dado el carácter multifactorial de la muerte embrionaria temprana recurrente. Lo que se puede concluir es que las alteraciones de las pruebas analizadas, pueden contribuir a la disminución de la fertilidad en parejas con historia de muerte embrionaria temprana recurrente.

\section{Conflicto de intereses y financiación}

Todos los autores de este trabajo declaramos que no existe conflicto de intereses.

El proyecto fue financiado por el CODI-Universidad de Antioquia; Aura María Gil-Villa y Walter Cardona-Maya fueron financiados por becas de Colciencias.

\section{Referencias}

1. Agarwal A, Saleh RA, Bedaiwy MA. Role of reactive oxygen species in the pathophysiology of human reproduction. Fertil Steril. 2003;79:829-43.

2. Evenson DP, Jost LK, Marshall D, Zinaman MJ, Clegg $\mathrm{E}$, Purvis $\mathrm{K}$, et al. Utility of the sperm chromatin structure assay as a diagnostic and prognostic tool in the human fertility clinic. Hum Reprod. 1999;14:1039-49.

3. Agarwal A, Makker K, Sharma R, Ashok Agarwal. Clinical relevance of oxidative stress in male factor infertility: An update. Am J Reprod Immunol. 2008;59:2-11.

4. Koca Y, Ozdal OL, Celik M, Unal S, Balaban NY. Antioxidant activity of seminal plasma in fertile and infertile men. Arch Androl. 2003;49:355-9.

5. Aitken RJ, Clarkson JS, Fishel S. Generation of reactive oxygen species, lipid peroxidation, and human sperm function. Biol Reprod. 1989;41:183-97.

6. Agarwal A. Significance of oxidative stress and sperm chromatin damage in male infertility. In: De Vriese S. Christophe A, editors. Male fertility and lipid metabolism. Champaign, IL: AOCS Press; 2003. p.157. 
7. Rubio C, Simon C, Blanco J, Vidal F, Minguez Y, Egozcue $\mathrm{J}$, et al. Implications of sperm chromosome abnormalities in recurrent miscarriage. J Assist Reprod Genet. 1999;16:253-8.

8. Godpalkrishnam K, Padwal V, Mejherji PK, Gokral JS, Shan R, Juneja HS. Poor quality of sperm as it affects repeated early pregnancy loss. Arch Androl. 2000;45:111-7.

9. Bernardini LM, Costa M, Botazzi C, Gianaroli L, Magli MC, Venturini PL, et al. Sperm aneuploidy and recurrent pregnancy loss. Reprod Biomed Online. 2004;9:312-20.

10. Molina J, Castilla JA, Castano JL, Fontes J, Mendoza $\mathbf{N}$, Martínez L. Chromatin status in human ejaculated spermatozoa from infertile patients and relationship to seminal parameters. Hum Reprod. 2001;16:534-9.

11. Sharma RK, Pasqualotto FF, Nelson DR, Thomas AJ. The reactive oxygen species-total antioxidant capacity score is a new measure of oxidative stress to predict male infertility. Hum Reprod. 1999;14:2801-7.

12. Gil-Villa AM, Cardona-Maya W, Agarwal A, Sharma R, Cadavid A. Role of male factor in early recurrent embryo loss: Do antioxidants have any effect? Fertil Steril. 2009;92:565-71.

13. Gil-Villa AM, Cardona-Maya WD, Agarwal A, Sharma R, Cadavid AP. Assesment of sperm factors possibly involved in early recurrent pregnancy loss. Fertil Steril. 2010;94:146572.

14. Gil-Villa A, Cardona-Maya W, Cadavid A. Muerte embrionaria temprana: ¿tiene influencia el factor masculino? Arch Esp Urol. 2007;60:1057-68.

15. World Health Organization. Laboratory manual for the examination of human semen and sperm-cervical mucus interaction. Cambridge, U.K.: Cambridge University Press; 1999.

16. Kruger TF, Menkeveld R, Stander FS, Lombard CJ, van Der Merwe JP, van Zyl JA, et al. Sperm morphologic features as a prognostic factor in vitro fertilization. Fertil Steril. 1986;46:1118-23.

17. Departamento de Estadística. Universidad Carlos III de Madrid. Análisis Discriminante. Última Actualización Junio 2009. Fecha de consulta: junio de 2009. Disponible en: http://halweb.uc3m.es/esp/Personal/personas/jmmarin/ esp/AMult/tema6am.pdf.
18. Agarwal A, Prabakaran SA. Mechanism, measurement, and prevention of oxidative stress in male reproductive physiology. Indian J Exp Biol. 2005;43:963-74.

19. Chi HJ, Kim JH, Ryu CS, Lee JY, Park JS, Chung DY, et al. Protective effect of antioxidant supplementation in sperm-preparation medium against oxidative stress in human spermatozoa. Hum Reprod. 2008;23:1023-8.

20. Saleh RA, Agarwal A. Oxidative stress and male infertility: From research bench to clinical practice. J Androl. 2002;23:737-52.

21. Lopes S, Jurisicova A, Sun Jg, Casper RF. reactive oxygen species: potential cause for DNA fragmentation in human spermatozoa. Hum Reprod. 1998;13:896-900.

22. Potts RJ, Notarianni LJ, Jefferies TM. Seminal plasma reduces exogenous oxidative damage to human sperm, determined by the measurement of DNA strand breaks and lipid peroxidation. Mutat Res. 2000;447:249-56.

23. Agarwal A, Said TM. Role of sperm chromatin abnormalities and DNA damage in male infertility. Hum Reprod Update. 2003:9:331-45.

24. Shamsi MB, Kumar R, Dada R. Evaluation of nuclear DNA damage in human spermatozoa in men opting for assisted reproduction. Indian J Med Res. 2008;127:115-23.

25. Carrell DT, Liu L, Peterson CM, Jones KP, Hatasaka HH, Erickson L, et al. Sperm DNA fargmentation is increased in couples with unexplained recurrent pregnancy loss. Arch Androl. 2003;49:49-55.

26. Khosrowbeygi A, Zarghami N. Levels of oxidative stress biomarkers in seminal plasma and their relationship with seminal parameters. BMC Clin Pathol. 2007;7:6.

27. Twigg J, Fulton, N, Gómez E, Irvine DS, Aitken RJ. Analysis of the impact of intracellular reactive oxygen species generation on the structural and functional integrity of human spermatozoa: Lipid peroxidation, DNA fragmentation and effectiveness of antioxidants. Hum Reprod. 1998;13:1429-36.

28. Keskes-Ammar L, Feki-Chakroun N, Rebai T, Sahnoun Z, Ghozzi H, Hammami S, et al. Sperm oxidative stress and the effect of an oral vitamin $E$ and selenium supplement on semen quality in infertile men. Arch Androl. 2003;49:83-94 